\title{
Quantum Dynamics of a Dissipative Object in a Periodic Potential.
}

\author{
U. ECKERN and F. PELzer \\ Institut für Theorie der Kondensierten Materie, Universität Karlsmihe, \\ Postfach 6980 D-7500 Karlsruhe 1
}

PACS. 03.65. - Quantum theory; quantum mechanics.

PACS. 05.40. - Fluctuation phenomena, random processes, and Brownian motion.

\begin{abstract}
The dynamics of a quantum particle moving in a periodic potential and coupled to a dissipative environment is investigated. Field-theoretical methods as introduced by Keldysh are used to determine the generating functional for real-time finite-temperature correlation functions. Recent results for the weak corrugation limit are confirmed, and extended, by summing an infinite set of higher-order terms, which allows us to express the mobility in terms of the self-energy. The relation to recent experiments on the superconductivity of granular films is discussed.
\end{abstract}

Stimulated by the ideas of Leggett [1] on the consequences of quantum mechanics when applied to a macroscopic object, and by the investigation of Caldeira and Leggett of macroscopic quantum tunnelling [2], several authors have studied the problem of quantum coherence for a particle coupled to a dissipative environment [3-7]. Surprisingly, the behaviour depends critically on the value of the friction constant, $\eta$. Consider, for example the dynamics of an object in a periodic potential $V(q)=-g \cos q$, where $q$ is the (dimensionless) co-ordinate, whose classical equation of motion is given by $\left({ }^{1}\right)$

$$
m \ddot{q}+\eta \dot{q}=-g \sin q+F
$$

where $F=F(t)$ is an external force. Apparently, for $g=0$, the (normalized) mobility is given by $\mu_{0}(\omega)=i \omega \eta /\left(m \omega^{2}+i \omega \eta\right)$; especially $\mu_{0}(0)=1$. On the other hand, for $g$ finite, the classical treatment shows that $\mu(0) \rightarrow 0$ for $T \rightarrow 0$, where $T$ is the temperature. However, considering the quantum dynamics of this model [4-6], a diffusion-localization transition is found at zero temperature, upon increasing $\alpha=2 \pi \eta$ through its critical value $\alpha=1$. Correspondingly, $\mu(0)=1$ or $\mu(0)=0$, for $\alpha<1$ or $\alpha>1$, respectively. In addition, as a function of temperature, the mobility decreases and increases again upon lowering $T$, for $\alpha<1$, while it decreases

(i) Units are chosen such that $g m^{-1}$, and $F$ are in units of frequency. Thus, strictly speaking, $\hbar \cdot V(q)$ is the potential energy. 
monotonically to zero for $\alpha>1$ [6]. Qualitatively, such a behaviour was observed in recent measurements of the resistance of granular Sn films [8]. (See also [9, 10], and below.)

In this article, we study the quantum dynamics of an object («particle») whose classical equation of motion is given by (1), by means of a field-theoretical approach due to KELDYSH [11]. This technique $\left({ }^{2}\right)$ has conceptual as well as computational advantages compared to other approaches, in particular, standard perturbation theory can be employed.

As an important feature of the Keldysh technique, there appears a contour, say $C$, with respect to time integration which runs from $t=-\infty$ to $t=+\infty$ (forward path; path index $\beta=1$ ) and from $t=+\infty$ to $t=-\infty$ (backward path; path index $\beta=2$ ). Accordingly, we wish to specify whether the time argument of a Heisenberg operator, for example, $\hat{q}(t)$, is on the forward or backward path. Thus, at a given time, we have formally two operators, $\hat{q}_{1}(t)$ and $\hat{q}_{2}(t)$, which can be combined to a vector $\hat{q}(t)=\left(\hat{q}_{1}(t), \hat{q}_{2}(t)\right)$; it is also convenient to introduce $\hat{x}_{1}=\left(\hat{q}_{1}-\hat{q}_{2}\right) / \sqrt{2}, \hat{x}_{2}=\left(\hat{q}_{1}+\hat{q}_{2}\right) / \sqrt{2}$, and $\hat{\boldsymbol{x}}=\left(x_{1}, x_{2}\right)$. Then we define the generating functional

$$
Z[\zeta(t)]=\left(\left(T_{C} \exp \left[i \int_{-\infty}^{\infty} \mathrm{d} t \zeta(t) \cdot \hat{x}(t)\right]\right)\right.
$$

where $T_{C}$ orders in time along the contour $C$, and the expectation value is with respect to the equilibrium statistical operator. This means that the system (object and environment) has been in thermal equilibrium in the infinite past. Note that, by this assumption, our approach differs from the Feynman-Vernon theory [14], which employs a somewhat artificial factorization of the density matrix at an initial time. The mobility, $\mu(\omega)$, is obtained from (2) by the following relation:

$$
\mu(\omega)=i \omega \eta \cdot\left\{\left.i \frac{\delta^{2} Z[\zeta]}{\delta \zeta_{2}(t) \delta \zeta_{1}\left(t^{\prime}\right)}\right|_{\zeta=0}\right\}
$$

where \{\}$_{\omega}$ denotes the Fourier transform with respect to the time difference.

Applying the perturbation theory with respect to the potential, we write

$$
Z[\zeta]=\left\{\exp \left[i t_{\text {int }}\left[-i \frac{\delta}{\partial \zeta}\right]\right]\right\} Z_{0}[\zeta]
$$

where $t_{\text {int }}$ (the subscript refers to interaction») is given by [12]

$$
\mathcal{A}_{\text {int }}[x]=-\int_{-\infty}^{\infty} \mathrm{d} t\left[V\left(q_{1}(t)\right)-V\left(q_{2}(t)\right)\right]=-2 g \int_{-\infty}^{\infty} \mathrm{d} t \sin \left(x_{1} / \sqrt{2}\right) \sin \left(x_{2} / \sqrt{2}\right) .
$$

The harmonic generating functional, $Z_{0}[\zeta]$, is of the standard Gaussian form, namely

$$
Z_{0}[\zeta]=\exp \left[-\frac{i}{2} \zeta \zeta\left|D_{0}\right| \zeta \supset\right]
$$

where

$$
c \zeta D_{0} \mid \zeta \supset=\sum_{\beta, \beta^{\prime}=1}^{2} \int \mathrm{d} t \mathrm{~d} t^{\prime} \xi_{\beta}(t) D_{0}^{\beta^{\prime}}\left(t, t^{\prime}\right) \xi_{\beta^{\prime}}\left(t^{\prime}\right)
$$

(2) Some details of the Keldysh technique are given in [12,13]. 
The $2 \times 2$ matrix Green's function $D_{0}$ of the harmonic problem has elements given by $D_{0}^{11}=0, D_{0}^{12}=D_{0}^{\mathrm{A}}, D_{0}^{21}=D_{0}^{\mathrm{R}}, D_{0}^{22}=D_{0}^{\mathrm{K}}$, where $\mathrm{R}(\mathrm{A})$ denotes the retarded (advanced) function, and $\mathrm{K}$ the Keldysh component. The Fourier transforms of these quantities are given by $D_{0}^{\mathrm{R}}(\omega)=\left(m \omega^{2}+i \omega \eta\right)^{-1}, D_{0}^{\mathrm{A}}(\omega)=\left[D_{0}^{\mathrm{R}}(\omega)\right]^{*}$ and, from the fluctuation-dissipation theorem, $D_{0}^{\mathrm{K}}(\omega)=\left[D_{0}^{\mathrm{R}}(\omega)-D_{0}^{\mathrm{A}}(\omega)\right] \operatorname{ctgh}(\hbar \omega / 2 k T)$. We immediately verify the usual results for the harmonic case; for example, the mobility is given by $\mu_{0}(\omega)=i \omega n D_{0}^{\mathrm{R}}(\omega)$. Note that the external field can be included in (6) by the replacement $\zeta_{1} \rightarrow \zeta_{1}+\sqrt{2} F$.

It is convenient to rewrite (5) in terms of "charges $\delta= \pm 1, \varepsilon= \pm 1$, such that

$$
\mathscr{A}_{\text {int }}[x]=\frac{g}{2} \sum_{i, s= \pm 1} \int_{-\infty}^{\infty} d t \delta \varepsilon \exp \left[i\left(\delta x_{1}+\varepsilon x_{2}\right) / \sqrt{2}\right]
$$

Introducing «charge densities $\boldsymbol{\rho}(t)=\left(p_{1}(t), p_{2}(t)\right)$,

$$
\rho^{(n)}(t)=\frac{1}{\sqrt{2}} \sum_{k=1}^{n}\left(\begin{array}{l}
\delta_{k} \\
\varepsilon_{k}
\end{array}\right) \delta\left(t-t_{k}\right)
$$

we obtain in a first step

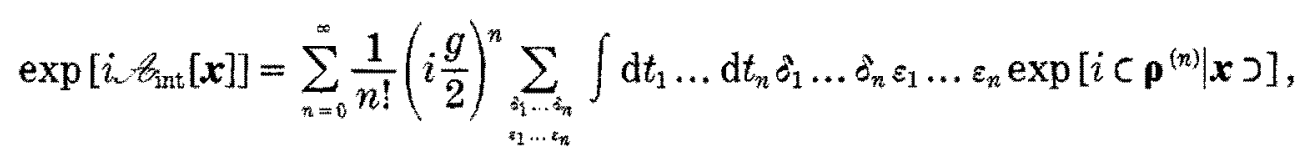

where $C \boldsymbol{\rho} \mid \boldsymbol{x} \supset=\int \mathrm{d} t \boldsymbol{\rho}(t) \cdot \boldsymbol{x}(t)$. This equation is written as $\exp \left[i f_{\text {int }}[\boldsymbol{x}]\right]=\operatorname{Tr} \exp$. $\cdot[i \subset \rho \mid x \supset]$, which defines *Tr» by comparison with (9). Inserting this result into (4) and applying Taylor's formula, we obtain

$$
Z[\zeta]=\operatorname{Tr} Z_{0}[\zeta+\rho]
$$

As a general property of the Keldysh technique, we remark that $Z[0]=\operatorname{Tr} Z_{0}[\rho]=1$; thus the normalization of the generating functional is preserved in the presence of the interaction. This can be shown as follows. Consider the term of order $n>0$ and focus on the part of the expression depending on d-charges:

$$
\sum_{\delta_{1} \ldots \delta_{n}} \delta_{1} \ldots \delta_{n} \exp \left[-i c_{p_{2}}\left|D_{0}^{R}\right|_{p_{1}} \supset\right]
$$

Here, the exponential is a part of $Z_{0}[\rho]$, using $C \rho\left|D_{0}\right| \rho \supset=c_{\rho_{2}}\left|D_{0}^{\mathrm{K}}\right|_{\rho_{2}} \supset+2 c_{\rho_{2}}\left|D_{0}^{\mathrm{R}}\right|_{\rho_{1}} \supset$. We insert $\rho(t)$, and perform the sum over $\delta$-charges, which leads to a product of sine-functions. Then, for a given ordering of the time arguments, we concentrate on the earliest time, $t_{k}<\left\{t_{i}\right\}_{i \neq k}$, and the factor resulting from the sum over $\delta_{k}$. Due to causality, this factor and thus the total expression is zero, leaving only the $n=0$ contribution, which gives $\operatorname{Tr} Z_{0}[\rho]=1$.

We consider in more detail the perturbation series of the connected Green's function, $\boldsymbol{D}$, which is defined by $\left(^{3}\right)$

$$
D^{\beta \beta^{\prime}}\left(t, t^{\prime}\right)=\left.i \frac{\delta^{2} \ln Z[\zeta]}{\partial \zeta_{\beta}(t) \delta \zeta_{\beta^{\prime}}\left(t^{\prime}\right)}\right|_{\zeta=0}
$$

(3) From now on, the external field will be included in $Z_{0}[\rho]$ by $\rho_{1} \rightarrow \rho_{1}+\sqrt{2} F$. 
Inserting (10) and defining an expectation value $\langle\cdot\rangle$ by

$$
\langle\cdot\rangle=\operatorname{Tr}\left(\cdot Z_{0}[\rho]\right),
$$

the following result is found:

$$
D=D_{0}+D_{0} S D_{0}
$$

with

$$
S^{\beta \beta^{\prime}}\left(t, t^{\prime}\right)=-i\left\langle\rho_{\beta}(t)_{\rho^{\prime}}\left(t^{\prime}\right)\right\rangle
$$

By arguments similar to those used to prove the normalization, we find $S^{22} \equiv 0$. Given an explicit expression for $\boldsymbol{S}$ in terms of a power series in $g$, we can compute the self-energy, $\boldsymbol{\Sigma}$, which contains only one-particle-irreducible diagrams, according to the relation

$$
\Sigma=S\left(1-D_{0} \Sigma\right)
$$

In terms of the self-energy, the mobility $\mu(\omega)=i \omega D^{\mathrm{R}}(\omega)\left(D^{\mathrm{R}} \equiv D^{21}\right)$ is given by

$$
\mu(\omega)=i \omega \eta\left[m \omega^{2}+i \omega \eta-\Sigma^{\mathrm{R}}(\omega)\right]^{-1}
$$

Turning to specific results, we first discuss the self-energy in first order in the coupling constant, $g$. We obtain

$$
\Sigma^{\mathrm{R}}=g \exp \left[-\frac{i}{4} D_{0}^{\mathrm{K}}(t=0)\right]+O\left(g^{2}\right)
$$

However, since $i D_{0}^{\mathrm{K}}(t=0)=+\infty$, the first-order term vanishes $\left({ }^{4}\right)$. Similarly, considering for $n>1$ the expression $\left.i C_{\rho_{2}}\left|D_{0}^{\mathrm{K}}\right| \rho_{2}\right)$, we conclude that this quantity is $+\infty$, unless

$$
\sum_{k=1}^{n} \varepsilon_{k}=0
$$

Thus only charge-neutral $\varepsilon$-charge configurations contribute to $\boldsymbol{S}$ (and $\boldsymbol{\Sigma}$ ). In particular, $n$ has to be even, i.e. $\Sigma$ is expressed as a power series in $g^{2}$. Considering the first term, we obtain from (15) and (16)

$$
\Sigma\left(t, t^{\prime}\right)=-i\left(g^{2} / 16\right) \sum_{\partial_{1}, \hat{\varepsilon}_{2}, t_{1}+\varepsilon_{2}=0} \int \mathrm{d} t_{1} \mathrm{~d} t_{2} \delta_{1} \partial_{2} Z_{0}[\rho] \sum_{k, l=1}^{2} \delta\left(t-t_{k}\right) \partial\left(t_{l}-t^{\prime}\right)\left\{\begin{array}{cc}
\partial_{k} \partial_{l} & \partial_{k} \varepsilon_{l} \\
\delta_{l} \varepsilon_{k} & 0
\end{array}\right\},
$$

$\left.{ }^{4}\right)$ One might be tempted to use the first-order expression for the self-energy to determine $\Sigma^{R}$ selfconsistently, which is clearly identical to the variational ansatz $[6,10]$. Thus, for $T=0$ and small $g$, one finds that $\alpha=1$ separates the diffusion from the localization regime. However, we also find a firstorder transition for $\alpha<1$ upon increasing $g$, similar to [10], but in disagreement with other investigations [5]. 
where we used $\varepsilon_{1} \varepsilon_{2}=-1$. Note that $\Sigma^{11}=\Sigma^{\mathrm{K}}, \Sigma^{12}=\Sigma^{\mathrm{R}}$ and $\Sigma^{21}=\Sigma^{\mathrm{A}}$. Explicitly, $Z_{0}[\rho]$ is given by

$$
Z_{0}[\rho]=\exp \left[\frac{\dot{i}}{2}\right]\left\{\hat{\delta}_{1} \varepsilon_{1} D_{0}^{\mathrm{A}}\left(t_{1}-t_{2}\right)-\partial_{2} \varepsilon_{1} D_{0}^{\mathrm{R}}\left(t_{1}-t_{2}\right)+D_{0}^{\mathrm{K}}\left(t_{1}-t_{2}\right)-D_{0}^{\mathrm{K}}(0)\right\}
$$

In the presence of a constant external field, $F$, the r.h.s. of (21) is multiplied by $\exp \left[i \varepsilon_{1} F\left(t_{1}-t_{2}\right) / \eta\right]$; thus the self-energy depends only on the time difference. We obtain the following result:

$$
\Sigma^{\mathrm{R}}(t)=g^{2} \sin \frac{D_{0}^{\mathrm{R}}(t)}{2} \cos \frac{F t}{\eta} \exp \left[\frac{i}{2}\left[D_{0}^{\mathrm{K}}(t)-D_{0}^{\mathrm{K}}(0)\right]\right]-\partial(t) B
$$

where the constant $B$ is given such that $\Sigma^{R}(\omega=0)=0$. For the Keldysh component, we find

$$
\Sigma^{\mathrm{K}}(t)=-i g^{2} \cos \frac{D_{0}^{\mathrm{R}}(t)}{2} \cos \frac{D_{0}^{\mathrm{A}}(t)}{2} \cos \frac{F t}{\eta} \exp \left[\frac{i}{2}\left[D_{0}^{\mathrm{K}}(t)-D_{0}^{\mathrm{K}}(0)\right]\right]
$$

Consequently, due to the factor $\cos (F t / \eta)$ in (22) and (23), we obtain the following relations for the Fourier transforms of $\Sigma^{\mathrm{R}}$ and $\Sigma^{\mathrm{K}}$ :

$$
\begin{gathered}
2 \Sigma^{\mathrm{R}}(\omega, F)=\left[\Sigma^{\mathrm{R}}(\omega+F / \eta, 0)+\Sigma^{\mathrm{R}}(\omega-F / \eta, 0)\right]-[\omega=0], \\
2 \Sigma^{\mathrm{K}}(\omega, F)=\Sigma^{\mathrm{K}}(\omega+F / \eta, 0)+\Sigma^{\mathrm{K}}(\omega-F / \eta, 0) .
\end{gathered}
$$

Finally, we consider in more detail the case $F=0$ and define the frequency-dependent correction of the friction constant, $\bar{\eta}(\omega)$, by the relation $-2 i \omega \cdot \bar{\eta}(\omega)=\Sigma^{\mathrm{R}}(\omega)-\Sigma^{\mathrm{A}}(\omega)$. Using $\Sigma^{\mathrm{K}}(\omega)=\left[\Sigma^{\mathrm{R}}(\omega)-\Sigma^{\mathrm{A}}(\omega)\right] \operatorname{ctgh}(\hbar \omega / 2 k t)$, which is a direct consequence of $(22)$ and (23), we arrive at $\bar{\eta}(\omega)=i \Sigma^{\mathrm{K}}(\omega) \operatorname{tgh}(\hbar \omega / 2 k T) / 2 \omega$. Thus we obtain the following expressions:

$$
\bar{\eta}(\omega)=\frac{g^{2}}{\omega} \int_{0}^{\infty} d t \sin \omega t \sin \left[\frac{\pi}{\alpha}(1-\exp [-\gamma t])\right] \exp \left[-\frac{2}{\alpha} f(t)\right]
$$

and

$$
\bar{\eta}(\omega)=\frac{g^{2}}{\omega} \operatorname{tgh}\left(\frac{\omega}{2 T}\right) \int_{0}^{\infty} \mathrm{d} t \cos \omega t \cos \left[\frac{\pi}{\alpha}(1-\exp [-\gamma t])\right] \exp \left[-\frac{2}{\alpha} f(t)\right],
$$

where we inserted $\left.D_{0}^{\mathrm{R}}(t)=D_{0}^{\mathrm{A}}(-t)=-\theta(t)\right][1-\exp [-\gamma t]] / \eta$ with $\gamma=\eta / m$; note that $\alpha=2 \pi \eta$. The quantity $f(t)$, given by

$$
f(t)=\int_{0}^{\infty} \frac{\mathrm{d} v}{\nu} \frac{\gamma^{2}}{v^{2}+\gamma^{2}} \operatorname{ctgh}\left(\frac{\hbar v}{2 k T}\right)(1-\cos v t),
$$

increases monotonically from $f(t=0)=0$ to infinity for increasing $t$. In particular, for $k T \ll \hbar \gamma$ and $\gamma t \geqslant 1$, we can approximate $f(t)$ by noting that the $\nu$-integration in (28) is cut off 
at $\vee \sim \gamma$; thus $f(t)=\ln [\sinh (\pi k T t / h) \hbar \gamma / \pi k T]$. This leads to the following result:

$$
\bar{\eta}(0) \simeq\left(\frac{\pi}{2} \exp [-2 C]\right)\left(\frac{g}{\gamma}\right)^{2}\left(\frac{k T}{h_{\gamma}}\right)^{2 / z-2} \quad \text { for } k T \ll h_{\gamma}
$$

where a $\alpha$-dependent prefactor (which we find to be equal to unity for $\alpha=1$ ) was omitted; $C$ is Euler's constant. With $\mu(0)=\eta /[\eta+\bar{\eta}(0)]$, we find $\mu(0)=1$ and $\mu(0)=0$ for $\alpha<1$ and $\alpha>1$, respectively, in the limit $T \rightarrow 0$. As an illustration, the temperature dependence of $\mu(0)$ is shown in fig. 1, for $\alpha=0.6$ and $\alpha=1.5(\bar{\eta}(0)$ is computed numerically from (26); in both cases, we have taken $g=\gamma$ ). Remarkable is the nonmonotic dependence on $T$, for $\alpha<1$, in contrast to the monotic dependence for $\alpha>1$. Note that, due to the partial summation of higher-order terms, a finite result is obtained for the mobility, even for $\alpha>1, T \rightarrow 0$.

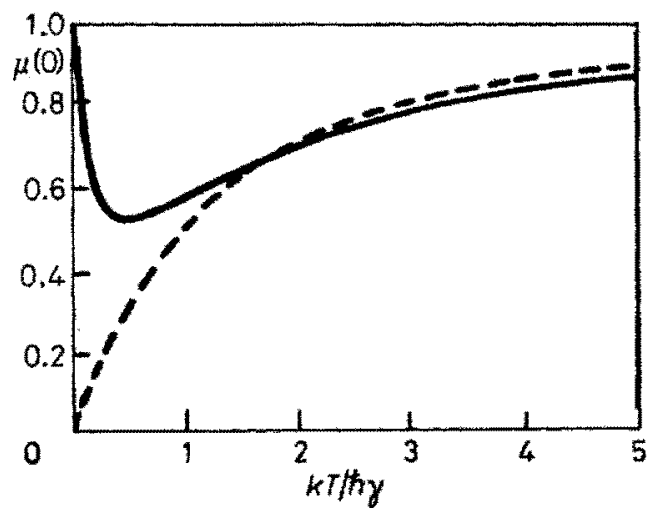

Fig. 1. - Normalized zero-frequency mobility, $\mu(\omega=0)$, as a function of temperature. The coupling constant is chosen to be $g=\gamma$. - $\alpha=0.6, \cdots \alpha=1.5$.

So far, we have concentrated on the weak corrugation limit. However, at zero temperature, there exists a duality transformation which relates the mobility for small $g$, i.e. $g \ll \omega_{0}$, where $\omega_{0}=(\mathrm{g} / \mathrm{m})^{\frac{1}{2}}$ is the frequency of classical small-amplitude oscillations, to the one in the opposite (tight binding) limit. To be precise, the partition function has been evaluated [4] by expansion with respect to the potential (method I), and by means of the instanton method (method II), respectively, the latter being asymptotically correct ${ }^{(5)}$ ) for $\omega_{0} \ll g$. For both cases, the result can be interpreted as the partition function of a neutral plasma. In addition, I and II can be mapped onto each other, provided the core region of the interaction is unimportant, i.e. for $T=0$ it is small compared to the average distance between charges: this leads to the conditions $\gamma^{-1} \ll g^{-1}$ (I) and $\omega_{0}^{-1} \ll \Delta^{-1}$ (II), respectively, where $\Delta$ is the tunnelling frequency. Then, for small frequencies, $\mu_{\mathrm{TB}}(\omega)=1-\tilde{\mu}(\omega)$, where $\tilde{\mu}(\omega)$ is obtained from $\mu(\omega)$ by using the replacement $\alpha \rightarrow \alpha^{-1}$, and $g / \gamma \rightarrow \Delta / \omega_{0}$. By similar arguments, the duality transformation is going to hold for finite temperatures, provided $h / k T$ (which is the length of the classical gas) is larger than the core of the interaction, i.e. $h \gamma \gg k T$ (I) and $\hbar \omega_{0} \gg k T$ (II), respectively; apparently, we need the additional rule $k T / \hbar \gamma \rightarrow k T / \hbar \omega_{0}$. For example, from (29), the zero frequency mobility in the tight-binding limit is found to be

(5) In addition, $\eta \leqslant g / \omega_{0}$, which includes the regime of very large damping, $\gamma \geqslant \omega_{0}$. 
given by

$$
\mu_{\mathrm{TB}}(0)=\frac{2 \pi \alpha \tilde{\eta}}{1+2 \pi \alpha \tilde{\eta}},
$$

where $\vec{\eta}=(\pi / 2)\left(\exp [-C] \Delta \omega_{0}\right)^{2}\left(k T / \hbar \omega_{0}\right)^{2(\alpha-1)}$. Consequently, for $T \rightarrow 0$, we obtain $\mu_{\mathrm{TB}}(0) \rightarrow 0$ for $\alpha>1$ and $\mu_{\mathrm{TB}}(0) \rightarrow 1$ for $\alpha<1$, which is the same behaviour as in the weak corrugation limit. Note that, for $\alpha=1$, we obtain $\mu_{\mathrm{TB}}(0)=\tilde{\Delta}^{2} /\left[1+\tilde{J}^{2}\right]$, where $\tilde{\Delta} \simeq \pi \Delta / \omega_{0}$ (see also [5a]). In fact, recent investigations of the tight-binding limit [15-17] lead to results in agreement with (3), except for the denominator, which in our expression is due to the use of the self-energy. As a consequence, our result gives a meaningful answer for $T \rightarrow 0$ even if $\alpha<1$.

The present model applies to a resistively shunted Josephson junction: the co-ordinate of the macroscopic object is identified with the phase difference across the junction, and the parameters are related by $m=\hbar C / 4 e^{2}, \alpha=R_{0} / R_{\text {s }}$ with $R_{0}=2 \pi \hbar / 4 e^{2} \simeq 6453 \Omega, \gamma=\left(R_{\mathrm{s}} \mathrm{C}\right)^{-1}$, and $g=I_{0} / 2 e$, where $C$ is the capacitance, $\mathrm{R}_{\mathrm{s}}$ the resistance of the shunt and $I_{0}$ the critical current. From the Josephson relation, $\hbar \dot{q}=2 \mathrm{eV}$, where $V$ is the voltage, it is apparent that the mobility of the object is proportional to the resistance of the junction. Indeed, recent experiments on granular Sn films [8] show a nonmonotonic temperature dependence of the resistance for $R_{\mathrm{N}} \geqslant 6 \mathrm{k} \Omega$, in contrast to a monotonic decrease for samples with $R_{\mathrm{N}} \leqslant 6 \mathrm{k} \Omega$, where $R_{\mathrm{N}}$ is the normal state sheet resistance, in qualitative agreement with the discussion above provided $R_{N}$ is identified with $R_{s}$. In short, the argument [9] is as follows: superconducting granular films are modelled as an array of superconducting islands connected by Josephson junctions, and this picture is combined with percolation arguments [18] which indicate that the resistance of the film is determined by the resistance of a single junction. (Thus, assuming a temperature-independent shunt resistance-which is not at all obvious-one identifies $R_{s}$ with the measured normal state resistance $R_{N}$.) However, quantitative theoretical investigations, for example of the temperature dependence of the resistance, and of realistic models, remain to be done. In particular, percolation and localization effects seem to play an important role $[19,20]$ and should be incorporated in a detailed theory.

Finally, we want to stress that the results obtained here and elsewhere $[4-6,9,10,16,17]$, rely on the model of ohmic dissipation, i.e. the presence of a shunt resistor. Physically, a shunt allows for a continuous charge transfer across the junction. On the other hand, if the dissipation is due to quasi-particle tunnelling [21], charge is transferred in units of the elementary charge [22]. In fact, in such a case, a qualitatively different picture of the motion in a periodic potential emerges [23]. The mechanism of dissipation in granular films seems to us an open question.

In conclusion, we have demonstrated that field-theoretical methods provide a transparent and straightforward approach to the dynamics of an object in a periodic potential, in the presence of ohmic dissipation and at finite temperatures. We emphasize that, in contrast to the Feynman-Vernon theory [14] (see also [6]), the present approach assumes that the system (object and environment) has been in thermal equilibrium in the infinite past. In particular, this means that we do not have to wait until transients arising from the factorization of the density matrix at an initial time have become unimportant. Thus we were able to consider directly frequency-dependent correlation functions, and we applied standard perturbation theory to compute the self-energy, with the result discussed above. We expect that the present techniques may be a convenient tool in other situations (like quasi-particle tunnelling) as well.

$$
* * *
$$

We would like to thank G. SCHöN, and especially A. SCHMID, for stimulating discussions. 


\section{REFERENCES}

[1] Leggett A. J., Progr. Theor. Phys., Suppl. 69 (1980) 80.

[2] Caldeira A. O. and Leggett A. J., Phys. Rev. Lett., 46 (1981) 211; A. Phys. (N.Y.), 149 (1988) $374 ; 153(1984)$ 445(E).

[3] Chakravarty S., Phys. Rev. Lett., 49 (1982) 681; Bray A. J. and Moore M. A., Phys. Rev. Lett., 49 (1982) 1545.

[4] SCHMId A., Phys. Rev. Lett., 51 (1983) 1506.

[5] a) Guinea F., Hakim V. and Muramatsu A., Phys. Rev. Lett., 54 (1985) 263; b) Guinea F., Phys. Rev. B, 32 (1985) 7518.

[6] Fisher M. P. A. and Zwerger W., Phys. Rev. B, 32 (1985) 6190.

[7] Leggett A. J., Chakravarty S., Dorsey A. T., Fisher M. P. A., Garg A. and Zwerger W., Dynamics of the Dissipative Two-State System, preprint.

[8] OrR B. G., Jaeger H. M., Goldman A. M. and Kuper C. G., Phys. Rev. Lett., 56 (1986) 378.

[9] Fisher M. P. A., Phys, Rev. Lett., 57 (1986) 885.

[10] Chakravarty S., INgold G.-L., Kivelson S. and Luther A., Phys. Rev. Lett., 56 (1986) 2303.

[11] Keldysh L. V., Z̆. Éksp. Teor. Fiz., 47 (1964) 1515 [Sov. Phys. JETP, 20 (1965) 1018].

[12] Schmid A., J. Low Temp. Phys., 49 (1982) 609.

[13] Schmid A., Repeated Measurements on Dissipative Linear Quantum Systems, preprint.

[14] Feynmann R. P. and Vernon F. L. jr., Ann. Phys. (N.Y.), 24 (1963) 118; see also: Feynman R. P. and HrBbs A. R., Quantum Mechanics and Path Integrals (McGraw-Hill, New York, N. Y.), 1965.

[15] Kondo J., Physica $B+C, 126$ (1984) 377.

[16] Weiss U. and Grabert H., Phys. Lett. A, 108 (1985) 63.

[17] Aslangul C., Pottier N. and Saint-James D., Phys. Lett. A, 111 (1985) 175.

[18] Ambegaokar V., Halperin B. I. and Langer J. S., Phys. Rev. B, 4 (1971) 2612.

[19] ORR B. G., JAeger H. M. and Goldman A. M., Phys. Rev. B, 32 (1985) 7586.

[20] White A. E., Dynes R. C. and Garno J. P., Phys. Rev. B, 33 (1986) 3549.

[21] Ambegaokar V., Eckern U. and Schön G., Phys. Rev. Lett., 48 (1982) 1745; Phys. Rev. B, 30 (1984) 6419; Larkin A. I. and Ovchinnikov N., Phys. Rev. B, 28 (1983) 6281.

[22] BEN-JACOB E., MoTTola E. and Schön G., Phys. Rev. Lett., 51 (1983) 2064; Schön G., Phys. Rev. $B, 32$ (1985) 4469.

[23] GuineA F. and Schön G., Europhys. Lett., 1 (1986) 585. 